\title{
Magnetic field dependent resonant light scattering by magnetic spheres in a magnetizable medium
}

\author{
Hem Bhatt \\ Shantilal Shah Engineering College, Sidsar, Bhavnagar. 364060, India \\ drhembhatt@gmail.com
}

PACS 47.65.Cb, 42.25.Fx, 42.68.Ay, 41.20.Jb

DOI 10.17586/2220-8054-2016-7-3-479-481

\begin{abstract}
The anisotropy factor is a parameter from which one can determine preferential forward and backward light scattering. In the present study, we have calculated anisotropy factor $\langle\cos \theta\rangle$ as a function of magnetic field for magnetic spheres in a magnetic medium. We have noticed resonances in $\langle\cos \theta\rangle$ which indicate preferential enhanced scattering in forward and backward directions. Anisotropy factor is analyzed for various size parameters. The study clearly indicates that scattering can be modulated by means of size parameter of magnetic spheres as well as magnetic field. We have further confirmed the result by studying forward and backward intensity as a function of magnetic field. Resonances noticed in the intensity are in good agreement with the previous argument. This observation can be useful for the magnetic field dependent directional scattering and novel magneto-photonic devices.
\end{abstract}

Keywords: mie resonances, scattering, magnetic colloids, magneto-photonics.

Received: 23 January 2016. Revised: 8 March 2016

\section{Introduction}

In the recent past, the high energy and working efficiency of photonic devices have attracted researchers to design and study materials in which light transport can be tuned by controlling some external parameters. Recently, a new frontier has also emerged with a goal of controlling light transport by means of interference in artificially engineered optical materials and metamaterials [1-6]. Remarkable progress has been noted in the fabrication of nanophotonic structures with many novel properties [7]. The disorder present and the magnetic tenability have made magnetically polarizable fluids preferable for many applications like optical switches, modulators, filters and gratings, etc. [8,9]. Light transport and optical study of resonant behavior have been studied in disordered magnetic media along with coherent backscattering and light localization [10-13]. But very few have used magnetic tuning of magnetic spheres to predict theoretical and experimental photonic effects [14-16]. Several groups are working on single and multiple scattering by magnetic scatterers in non-magnetic medium [17-19]. Our recent studies have shown some unusual light transport in ferrocolloids in presence of external magnetic field [20]. This study reflects effect of magnetic field Mie resonance by magnetic spheres dispersed in magnetic nanofluids and its impact on light transport in magnetic colloids.

\section{Theory}

We consider a magnetizable sphere of arbitrary size parameter $\alpha(=2 \pi r \lambda)$ with ' $r$ ' being the radius of the particle and $\lambda$ is free space wavelength of incident light and refractive index $m_{s}$ surrounded by a magnetizable medium like a ferrofluid. According to Mie theory, scattered intensities for the two orthogonalized linearly polarized states are given by $i_{1}=\left|S_{1}\left(m_{s}, \alpha, \theta\right)\right|^{2}$ and $i_{2}=\left|S_{2}\left(m_{s}, \alpha, \theta\right)\right|^{2}, i_{1}$ and $i_{2}$ correspond to the scattered field components, perpendicular and parallel to the scattering plane (i.e. plane formed by the direction of applied field and the propagation direction). The scattering coefficients $S_{1}\left(m_{s}, \alpha, \theta\right)$ and $S_{2}\left(m_{s}, \alpha, \theta\right)$ are defined by:

$$
\begin{aligned}
& \left.S_{1}\left(m_{s}, \alpha, \theta\right)=\sum_{n=1}^{\infty} \frac{2 n+1}{n(n+1}\right)\left[\alpha_{n} \pi_{n}(\cos \theta)+b_{n} \tau_{n}(\cos \theta)\right], \\
& S_{2}\left(m_{s}, \alpha, \theta\right)=\sum_{n=1}^{\infty} \frac{2 n+1}{n(n+1)}\left[\alpha_{n} \tau_{n}(\cos \theta)+b_{n} \pi_{n}(\cos \theta)\right],
\end{aligned}
$$

where Mie coefficients $a_{n}$ and $b_{n}$ for magnetic sphere are defined by [20]:

$$
\begin{aligned}
& a_{n}=\frac{\mu \Psi_{n}^{\prime}(\beta) \Psi_{n}^{\prime}(\alpha)-m \Psi_{n}^{\prime}(\beta) \Psi_{n}^{\prime}(\alpha)}{\mu \Psi_{n}^{\prime}(\beta) \zeta_{n}^{\prime}(\alpha)-m \Psi_{n}^{\prime}(\beta) \zeta_{n}^{\prime}(\alpha)}, \\
& b_{n}=\frac{m \Psi_{n}(\beta) \Psi_{n}^{\prime}(\alpha)-\mu \Psi_{n}^{\prime}(\beta) \Psi_{n}^{\prime}(\alpha)}{m \Psi_{n}^{\prime}(\beta) \zeta_{n}^{\prime}(\alpha)-\mu \Psi_{n}^{\prime}(\beta) \zeta_{n}^{\prime}(\alpha)},
\end{aligned}
$$


and $\pi_{n}(\cos \theta)$ and $\tau_{n}(\cos \theta)$ are the partial derivative of the Legendre polynomials and angle $\theta$ is the angle between the forward and scattering directions. $\mu_{s}$ and $m_{s}$ are respectively magnetic permeability and refractive index of the scatterers with respect to the surrounding medium. $\psi_{n}$ and $\zeta_{n}$ are respectively Riccati Bessel and Hankel functions. Prime denote derivative with respect to arguments. The arguments of Riccati-Bessel and Hankel functions are given by $\alpha=2 \pi r / \lambda$ and $\beta=\left(m_{s} \alpha\right)$.

In the present case however, the medium is magnetically active and both $\mu_{m}$ and $m_{s}$ may depend on the applied magnetic field. Ferrofluids usually contain nanomagnetic particles of magnetic oxides like $\mathrm{Fe}_{2} \mathrm{O}_{3}$ or $\mathrm{Fe}_{3} \mathrm{O}_{4}$ hence we shall assume that at optical frequencies $\mu_{m} \sim 1$. The refractive index of the ferrofluid may be expressed as $m_{f}=m_{\infty} \mathrm{L}(\xi)+m_{0}$, where $m_{0}$ is the refractive index at zero field and $m_{\infty}$ is the saturation value of the field dependent refractive index and the Langevin function $L(\xi)=\operatorname{coth}(\xi)-\frac{1}{\xi}$, with $\xi=\frac{\mu H}{k T}$ where $\mu$ is the magnetic moment of the monodomain nanomagnetic particles of ferrofluid, $H$ is the applied field; $k$ and $T$ are respectively the Boltzmann constant and absolute temperature of the medium [21]. Considering the ferrofluid as a magnetizable medium, the relative refractive index of the MMS will be given by $m_{M M S}=m_{s c a t} / m_{f}$. Since $m_{f}$ depends on $\xi=\frac{\mu H}{k T} ; m_{M M S}$ will also depend on $\mathrm{H}$. This will lead to the field dependence of the scattering parameters $a_{n}$, $b_{n}, S_{1}\left(m_{s}, \alpha, \theta\right), S_{2}\left(m_{s}, \alpha, \theta\right)$ and $i_{1}$ and $i_{2}$.

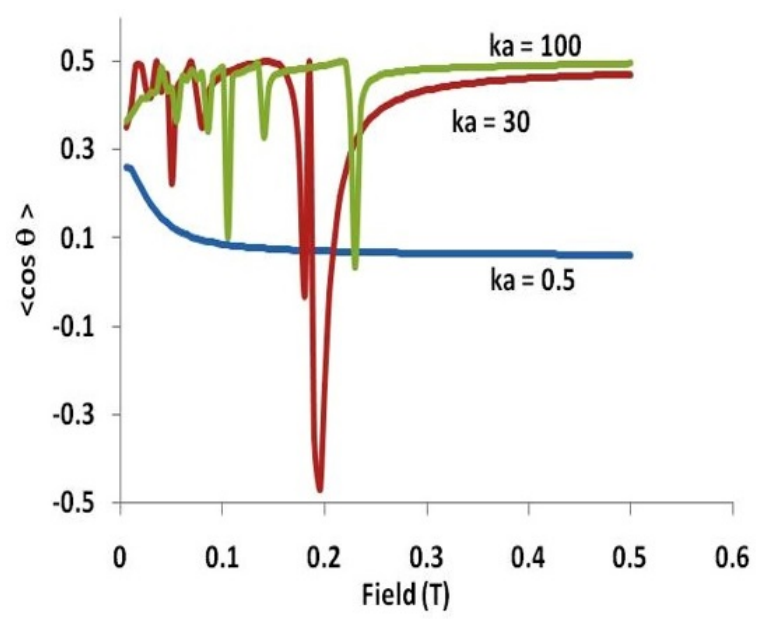

FIG. 1. It shows the $\langle\cos \theta\rangle$ as a function of magnetic field for different values of size parameter $k a$. For $k a=30$ large resonance is observed. The negative value suggests preferential backscattered intensity

\section{Result and discussion}

It is known that for micron sized dielectric spheres the incident light excites localized electromagnetic modes at the surface of spheres. Such modes may trap light near the surface of the sphere by repeated total internal reflections. Resonances in such systems are a consequence of the refractive index mismatch, or shape or size, and are called morphology-dependent resonance (MDR) [22].

In Fig. 1, the anisotropy factor $\langle\cos \theta\rangle$ is plotted as a function of the applied magnetic field for different size parameter $k a$. It is noted that for $k a=0.5$ no resonance is observed, it suggests that the system behaves as a Rayleigh scatterer. For $k a=30$ maximum resonant behavior is observed, but for $k a=100$ the resonance is not so strong. This suggests that for critical size parameter, the resonance is pronounced. The fact that $\langle\cos \theta\rangle$ can assume negative values indicates a predominant backward scattering. This is quite unusual in the case of scattering by non-magnetic spheres. The reason for the appearance of a preferential backscattering configuration in the small particle limit is the significant contribution given by magnetic dipolar radiation in magnetic scattering. These resonances may produce a standing wave configuration within the spheres that can cause the delay in light transport in magnetic colloids, which can be tunable by external magnetic field.

Figure 2 ( $\mathrm{A}$ and $\mathrm{B}$ ) shows the resonance produced in the light transport for $\sim 3 \mu \mathrm{m}$ size magnetic spheres suspended in magnetic nanofluids (for forward and backscattered light) as a function of magnetic field. It should be noted here that at certain magnetic field values, the transmitted intensity becomes zero and generates magnetically induced stop band of light. 

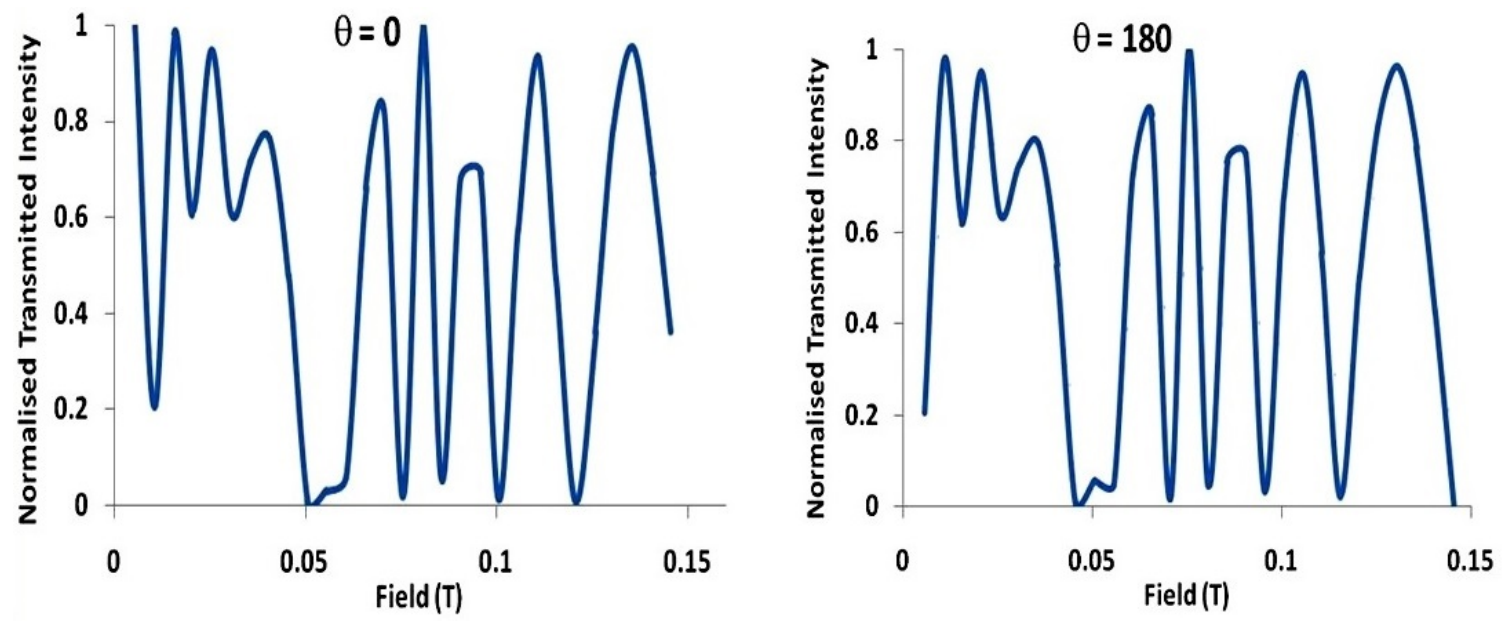

FIG. 2. Variation of forward and backward scattering intensity as functions of magnetic field for a magnetizable micronsized sphere (MMS) having diameter $3 \mu \mathrm{m}$ suspended in magnetic nanofluids. Transmitted intensity exhibits magnetic field dependent resonant behavior

\section{Conclusion}

The present study has shown that an externally applied magnetic field induced morphological dependent resonance in a magnetizable sphere surrounded by magnetic nanofluids. This system exhibits resonance in forward as well as in backward direction. $\langle\cos \theta\rangle$ as a function of magnetic field shows preferential backward scattering. Such resonance produces standing wave within the magnetic spheres and can cause delay in light transport. These properties of such a system are useful for the development of novel magneto-photonic devices.

\section{References}

[1] Yablonovitch E. Inhibited Spontaneous Emission in Solid-State Physics and Electronics. Phy. Rev. Lett., 1987, 58, P. $2059-2062$.

[2] John S. Strong localization of photons in certain disordered dielectric superlattices. Phys. Rev. Lett.,1987, 58, P. 2486.

[3] Anderson P.W. The question of classical localization A theory of white paint? Philos. Mag. B., 1985, 52, P. $505-509$.

[4] Kuga Y., Ishimaru A. Retroreflectance from a dense distribution of spherical particles. J. Opt. Soc. Am. A., 1984, 8, P. 831-835.

[5] Van Albada M.P., Lagendijk A. Observation of Weak Localization of Light in a Random Medium. Phy. Rev. Lett., 1985, 55, P. $2692-2695$.

[6] Sebbah P., Hu B., et al. Spatial-Field Correlation: The Building Block of Mesoscopic Fluctuations. Phys. Rev. Lett, 2002, 88, P. 123901-04.

[7] Sheng P. Introduction to Wave Scattering, Localization and Mesoscopic Phenomena. Academic, San Diago, 1995.

[8] Liu T., Chen X., et al. Tunable magneto-optical wavelength filter of long-period fiber grating with magnetic fluids. Appl. Phy. Lett., 2007, 91, P. 121116.

[9] Chieh J.J., Yang S.Y., et al. Magnetic-fluid optical-fiber modulators via magnetic modulation. Appl. Phy. Lett., 2007, 90, P. 133505.

[10] Pinheiro F.A., Martinez A.S., Sampaio L. Vanishing of energy of transport velocity and diffucsion constant of electromagnetic waves in disordered magnetic media. Phy. Rev. Lett., 2000, 85, P. 5563.

[11] Pinheiro F.A., Martinez A.S., Sampaio L. New Effects in Light Scattering in Disordered Media and Coherent Backscattering Cone: Systems of Magnetic Particles. Phy. Rev. Lett., 2000, 84, P. 1435-1438.

[12] Garcia P.D., Sapienza R., et al. Resonant light transport through Mie modes in photonic glasses. Phy. Rev. A., 2008, 78, P. 023823.

[13] Hau L.V., Harris S.E., Dutton Z., Behroozi C.H. Light speed reduction to 17 metres per second in an ultracold atomic gas. Nature, 1999, 397, P. 594.

[14] Kim H., Ge J., et al. Structural colour printing using a magnetically tunable and lithographically fixable photonic crystal. Nat. Photon., 2009, 3, P. 534-540.

[15] Pu S., Liu M. Tunable photonic crystals based on $\mathrm{MnFe}_{2} \mathrm{O}_{4}$ magnetic fluids by magnetic fields. J. Alloys and Comp., 2009, 481, P. 851-854.

[16] Ge J., Hu Y., Yin Y. Highly Tunable Superparamagnetic Colloidal Photonic Crystals. Angrew. Chem. Int. Ed., 2007, 46, P. 7428.

[17] Kerker M., Wang D.S., Giles C.L. Electromagnetic scattering by magnetic spheres. J. Opt. Soc. Am., 1983, 73(6), P. 765.

[18] García-Cámara B., Moreno F., et al. Light scattering resonances in small particles with electric and magnetic properties. J. Opt. Soc. Am., 2008, 25, P. 327-334.

[19] Tarento R.J. Mie Scattering of Magnetic Spheres. Phys. Rev. E, 2004, 69, P. 026606-1.

[20] Lacoste D., van Tigglen B.A. Coherent backscattering of light in a magnetic field. Phys. Rev. E, 2000, 61, P. 4556-4565.

[21] Chan Y.F., Yang S.Y., et al. Thermal effect on the field-dependent refractive index of the magnetic fluid film. Appl. Phys. Lett., 2003, 82, P. 3481-3484.

[22] Bhatt H., Patel R., Mehta R.V. Magnetically induced Mie resonance in a magnetic sphere suspended in a ferrofluid. J. Opt. Soc. Am A, 2010, 27, P. 873 . 